\title{
Difluoromethylornithine attenuates isoproterenol-induced cardiac hypertrophy by regulating apoptosis, autophagy and the mitochondria-associated membranes pathway
}

\author{
YU ZHAO*, WEI-WEI JIA* , SAN REN, WEI XIAO, GUANG-WEI LI, LI JIN and YAN LIN \\ Department of Pathophysiology, Qiqihar Medical University, Qiqihar, Heilongjiang 161006, P.R. China
}

Received October 9, 2020; Accepted April 28, 2021

DOI: $10.3892 /$ etm.2021.10302

\begin{abstract}
Myocardial hypertrophy is an independent risk factor of cardiovascular diseases and is closely associated with the incidence of heart failure. In the present study, we hypothesized that difluoromethylornithine (DFMO) could attenuate cardiac hypertrophy through mitochondria-associated membranes (MAM) and autophagy. Cardiac hypertrophy was induced in male rats by intravenous administration of isoproterenol (ISO; $5 \mathrm{mg} / \mathrm{kg} /$ day) for 1, 3,7 and 14 days. For DFMO treatment group, rats were given ISO $(5 \mathrm{mg} / \mathrm{kg} / \mathrm{day})$ for 14 days and $2 \%$ DFMO in their water for 4 weeks. The expression of atrial natriuretic peptide (ANP) mRNA,heart parameters, apoptosis rate, fibrotic area and protein expressions of cleaved caspase3/9, GRP75, Mfn2, CypD and VDAC1 were measured to confirm the development of cardiac hypertrophy, apoptosis and autophagy induced by ISO. ANP mRNA and MAM protein expression levels were assessed by reverse transcription-quantitative PCR and western blotting to evaluate hypertrophy and the effects of DFMO oral administration. The results demonstrated that heart parameters, ANP mRNA levels, fibrotic area and apoptosis rate were significantly increased in the heart tissue for ISO 7 and 14 day groups compared with the control group. Furthermore, treatment with DFMO significantly inhibited these indicators, and DFMO downregulated the MAM signaling pathway and upregulated the autophagy pathway in heart tissue compared with the ISO 14 day group. Overall, all ISO-induced changes analyzed in the present study were attenuated following treatment with DFMO. The findings form this study suggested that DFMO treatment may be considered as a potential strategy for preventing ISO-induced cardiac hypertrophy.
\end{abstract}

Correspondence to: Professor Yan Lin, Department of Pathophysiology, Qiqihar Medical University, 333 Bukui Street, Qiqihar, Heilongjiang 161006, P.R. China

E-mail: yanlinqqhr@aliyun.com

${ }^{*}$ Contributed equally

Key words: cardiac hypertrophy, difluoromethylornithine, mitochondria-associated membranes, apoptosis, autophagy

\section{Introduction}

The initial stage of cardiac hypertrophy is an adaptive response in volume related to hypertension, valvular disease or neurohumoral stimuli. The decompensated stage of pathological hypertrophy leads to contractile dysfunction and heart failure (HF) $(1,2)$. Mitochondria serve crucial biological roles in excitation-contraction coupling in cardiac muscle, cellular metabolism, HF progression and myocardial ischemia/reperfusion (3-5). It has been hypothesized that abnormalities in mitochondrial function and endoplasmic reticulum (ER) may be related to cardiomyopathy $(6,7)$.

Increasing evidence suggests that mitochondria and the ER are organelles that form an endomembrane network. Mitochondria-associated membranes (MAM) appear to be the contact point through which the ER directly communicates with mitochondria (8-10). The ER-mitochondria interface is enriched in various proteins, such as the macromolecular complex composed of voltage-dependent anion channels 1 (VDAC1), glucose regulated protein 75 (GRP75) and inositol 1,4,5-triphosphate receptor (IP3R), which regulate $\mathrm{Ca}^{2+}$ transfer from the ER to mitochondria $(11,12)$. The crosstalk between these organelle and MAM in cardiomyopathy remains unknown and requires further investigation.

Polyamines, such as putrescine, spermidine and spermine, are involved in cell proliferation and survival and in numerous biological and pathological processes, including apoptosis, regulation of Kir channels and oxidative stress (13-15). Previous studies have reported that elevated polyamine levels are associated with cardiac hypertrophy $(16,17)$. Certain stimuli, such as growth factors and oncogenes (hormones, Myc and Ras) can rapidly increase ornithine decarboxylase (ODC) protein level, which is a key enzyme involved in cellular polyamine biosynthesis $(18,19)$. Difluoromethylornithine (DFMO) is an ornithine analog that has previously been reported to deplete cellular polyamines through inhibition of ODC and to promote cardioprotection by attenuating apoptosis and the NO/cGMP-dependent protein kinase-1 pathway $(17,20,21)$. Subsequently, the present study investigated whether isoproterenol (ISO)-induced cardiomyopathy could be associated with increased apoptosis and MAM. In addition, this study examined if DFMO could be considered as an effective pharmacological method to attenuate ISO-induced cardiomyopathy and if this possible effect may be mediated by MAM signaling 
pathway regulation. In the present study, it was hypothesized that cardiac hypertrophy induced by ISO was associated with apoptosis and MAM and polyamine depletion by DFMO exerts a protective effect on cardiac hypertrophy.

\section{Materials and methods}

Chemicals and reagents. ISO (cat. no. 51-30-9) and DFMO (cat. no. 70052-12-9) were both purchased from Sigma-Aldrich; Merck KGaA. The TUNEL detection kit was purchased from Roche Diagnostics $\mathrm{GmbH}$. The primary antibodies against cleaved caspase-3/9, caspase-3/9, VDAC1, GRP75, cyclophilin D (CypD), mitofusin 2 (Mfn2), autophagy related 5 (Atg5) and Beclin1 were obtained from Santa Cruz Biotechnology, Inc. TRIzol reagent and a PrimeScript RT Reagent kit were from Takara Biotechnology. Protein extraction kit and GAPDH antibody (both Wuhan Boster Biological Technology, Ltd.) and Masson's trichrome staining kit (Beijing Solarbio Science \& Technology Co., Ltd.) were also purchased.

Animals. Healthy male Wistar rats weighing 200-250 g were used in the present study (Qiqihar Medicine University Animal Center). Rats were housed on a $12 \mathrm{~h}$ dark-light cycle at $23 \pm 1^{\circ} \mathrm{C}$. The study protocol was approved by the Institutional Animal Research Committee (approval no. QMU-AECC-2019-53).

Rat cardiac hypertrophy model and drug treatment. As described previously (15), rats were randomly divided into six groups ( $n=10$ per group) as follows: i) Control group, rats were given subcutaneous injections of $0.9 \%$ saline; ii) ISO1d group, rats were given ISO $(5 \mathrm{mg} / \mathrm{kg} /$ day $)$ for 1 day; iii) ISO3d group, rats were given ISO (5 mg/kg/day) for 3 days; iv) ISO7d group, rats were given ISO (5 mg/kg/day) for 7 days; v) ISO14d group, rats were given ISO ( $5 \mathrm{mg} / \mathrm{kg} /$ day) for 14 days; and vi) DFMO treatment group, rats were given ISO $(5 \mathrm{mg} / \mathrm{kg} /$ day) for 14 days and $2 \%$ DFMO in their water for 4 weeks. Following treatment, all rats were euthanized using $3 \%$ pentobarbital sodium $(80 \mathrm{mg} / \mathrm{kg})$ and hearts were collected for further experiments. The hearts were carefully isolated, excised and weighed in cold buffer. The ventricles were separated and weighed and the degree of ventricular hypertrophy was assessed by evaluating the heart-to-body weight $(\mathrm{HW} / \mathrm{BW})$ ratio and left ventricle-to-body weight (LVW/BW) ratio.

Quantification of fibrosis. Heart tissue samples were cut and fixed in $10 \%$ formalin for 2-3 days and embedded in paraffin at room temperature. To evaluate morphological changes, tissue samples were stained with Masson's trichrome reagent at room temperature for 120-160 min. A light microscope was used to visualize the cardiac sections at $\times 200$ magnification. The degree of collagen deposition was analyzed using Image-Pro Plus v6.0 (Media Cybernetics, Inc.). A total of $\geq 100$ myocardial cells in each group was analyzed.

Reverse transcription quantitative (RT-q)PCR. As previously described $(22,23)$, total RNA was isolated using TRIzol according to the manufacturer's instruction (Invitrogen; Thermo Fisher Scientific, Inc.) from left ventricular tissues at room temperature. A cDNA synthesis kit (Takara Biotechnology Inc.) and oligo(dT) primers were subsequently used to synthesize the first strand cDNA. RT-qPCR was performed using PowerUp SYBR-Green Master Mix and the results were analyzed using the StepOne ${ }^{\mathrm{TM}}$ (Thermo Fisher Scientific, Inc.) system with GAPDH as an internal control. The primers for ANP (a marker gene of cardiac hypertrophy) were as follows: forward 5'-GGGAAGTCAACCCGTCTCA-3' and reverse 5'-GGGCTC CAATCCTGTCAAT-3'. The primers for GAPDH were as follows: Forward, 5'-GAGACAGCCGCATCTTCTTG-3' and reverse, 5'-ATACGGCCAAATCCGTTCAC-3'. The thermocycling conditions were as follows: Initial denaturation at $95^{\circ} \mathrm{C}$ for $30 \mathrm{sec} ; 40$ cycles of annealing at $95^{\circ} \mathrm{C}$ for $5 \mathrm{sec}$ and extension at $60^{\circ} \mathrm{C}$ for $30 \mathrm{sec}$. The relative expression levels were normalized to endogenous control and were expressed as $2^{-\Delta \Delta \mathrm{Cq}}(20)$.

Western blotting. Harvested left ventricle tissues were frozen in liquid nitrogen and stored at $-80^{\circ} \mathrm{C}$ until prepared as described previously (19). Heart tissues were homogenized in RIPA lysis buffer containing protease and phosphate inhibitors on ice (cat. no. P0013B; Beyotime Institute of Biotechnology) and centrifuged at $12,000 \mathrm{x}$ g for $20 \mathrm{~min}$ at $4^{\circ} \mathrm{C}$. The protein concentration was determined using a BCA protein assay. Proteins samples were separated by electrophoresis in 10\% SDS-PAGE gels (20 $\mu \mathrm{g}$ protein/lane) and transferred onto PVDF membranes. After blocking with 5\% skimmed milk in Tris-buffered saline for $1.5 \mathrm{~h}$ at $25^{\circ} \mathrm{C}$, membranes were incubated with primary antibodies against GRP75 (1:1,000; cat. no. ab171089), VDAC1 (1:500; cat. no. ab14734), CypD (1:500; cat. no. ab231155), Mfn2 (1:500; cat. no. 11925S), GAPDH (1:1,000; cat. no. ab8245), cleaved caspase-3 (1:1,000; cat. no. $9661 \mathrm{~S})$, cleaved caspase-9 (1:1,000; cat. no. 20750S), Atg5 (1:500; cat. no. 2630S) and Beclin1 (1:500; cat. no. 3738S) overnight at $4^{\circ} \mathrm{C}$. The membrane was washed three times in TBST and incubated with horseradish peroxidase-conjugated secondary antibody (1:5,000; cat. no. BA1058; Wuhan Boster Biological Technology, Ltd.) in TBST for $1 \mathrm{~h}$ at $25^{\circ} \mathrm{C}$. The bands were detected using ECL chemiluminescence kit (cat. no. P0018M; Beyotime Institute of Biotechnology). Membranes were stripped with stripping buffer (cat. no. P0025; Beyotime Institute of Biotechnology) and re-probed with different antibodies. Relative expression levels were normalized to endogenous control GAPDH using ImageJ software (version 1.4.3.67; National Institutes of Health).

TUNEL staining. Cell apoptosis was assessed using TUNEL assay according to the manufacturers' instructions. The sections of heart tissue were cut into $6 \mu \mathrm{m}$ slices and then dewaxed. After xylene dewaxing and ethanol dehydration, the cardiac tissue sections were incubated with $20 \mu \mathrm{g} / \mathrm{ml}$ proteinase $\mathrm{K}$ for $20 \mathrm{~min}$ and fluorescein-labelled dUTP for $1 \mathrm{~h}$ at $37^{\circ} \mathrm{C}$. Fluorescence microscopy (x200 magnification; Olympus Corporation) was used to analyze $\geq 100$ myocardial cells in each group. Image-Pro Plus 6.0 (Media Cybernetics, Inc.) was used to calculate the results.

Immunohistochemical staining. Cardiomyocyte apoptosis was evaluated using caspase-3 and caspase-9 immunohistochemistry. Heart sections were cut into $4 \mu \mathrm{m}$ slices according to the protocol using the citric acid method (Citrate Antigen Retrieval Solution; cat. no. P0081; Beyotime Institute of Biotechnology). Sections were blocked with $8 \%$ goat serum for $30 \mathrm{~min}$ at $37^{\circ} \mathrm{C}$ and were incubated with caspase-3 (1:100; cat. no. ab13847, 

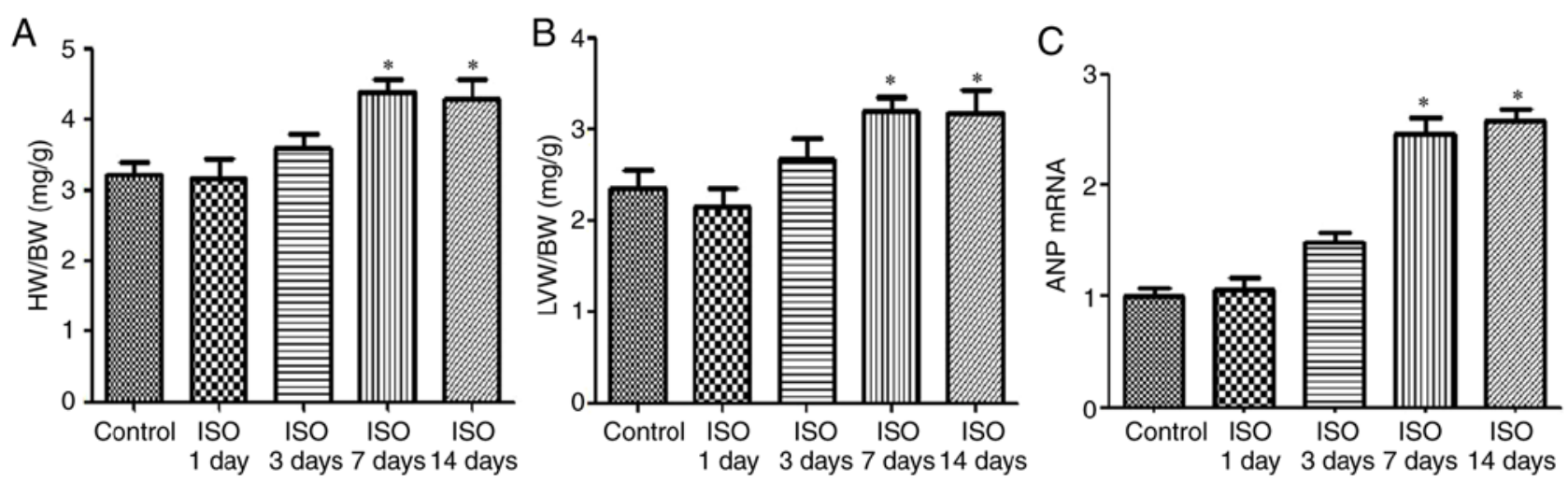

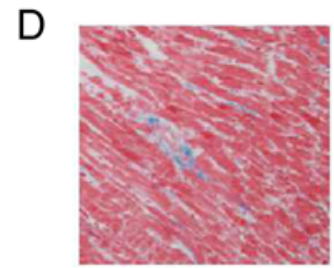

Control

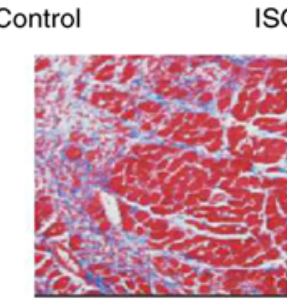

ISO 7 days

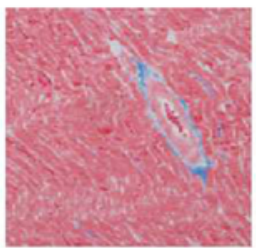

ISO 1 day

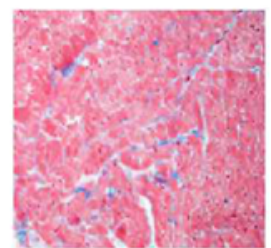

ISO 3 days

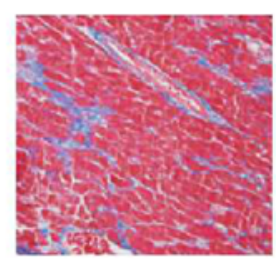

ISO 14 days

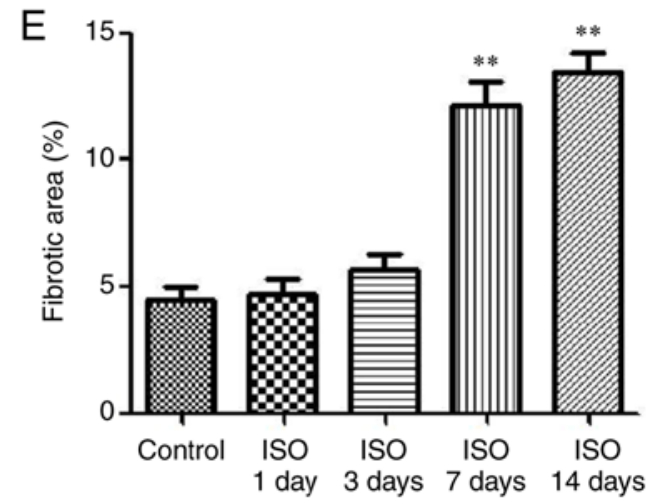

Figure 1. Hypertrophic state and interstitial fibrosis of heart tissue in control and ISO1, 3, 7 and 14d groups following ISO treatment. (A) HW/BW ratios. (B) LVW/BW ratios. (C) mRNA expression of ANP was detected by RT-qPCR. (D) Fibrosis of left ventricular tissue was detected by Masson staining (magnification, $\mathrm{x} 200$ ). Red staining indicates myocardial cells, and the interstitial fibers are stained as blue. (E) Quantification of fibrotic area. ${ }^{*} \mathrm{P}<0.05$ and ${ }^{* *} \mathrm{P}<0.01$ vs. control group. HW/BW, heart to body weight; LVW/BW, left ventricular to body weight; ANP, atrial natriuretic peptide; ISO, isoproterenol.

Santa Cruz Biotechnology, Inc.) and caspase-9 (1:100; cat. no. ab52298, Santa Cruz Biotechnology, Inc.) antibodies at $37^{\circ} \mathrm{C}$ for 2 h. EnVision ${ }^{\mathrm{TM}}+/ \mathrm{HRP}$ (cat. no. K400011-2; Dako; Agilent Technologies, Inc.) was added to sections for $1 \mathrm{~h}$ at $37^{\circ} \mathrm{C}$. Samples were processed using a peroxide-based substrate diaminobenzidine kit (Gene Tech Biotechnology Co., Ltd.) and nuclei were stained using haematoxylin for $1 \mathrm{sec}$ at room temperature. Finally, heart sections were photographed using a light microscope (magnification, x200). The results were analyzed using Image-Pro Plus 6.0 (Media Cybernetics, Inc.).

Statistical analysis. All data were presented as the means \pm standard error of the mean. One-way ANOVA followed by a Dunnett's or Student-Newman-Keuls post hoc tests were used for data analysis with GraphPad Prism 5.00 software (GraphPad Software, Inc.). $\mathrm{P}<0.05$ was considered to indicate a statistically significant difference.

\section{Results}

Effect of ISO on cardiac hypertrophy and cardiac fibrosis. To determine the effects of ISO on the development of cardiomyocyte hypertrophy, the cardiac hypertrophy model induced by ISO was established. Parameters of cardiac hypertrophy, such as $\mathrm{HW} / \mathrm{BW}$ ratio, $\mathrm{LVW} / \mathrm{BW}$ ratio, ANP mRNA expression were evaluated at 1, 3, 7 and 14 days following ISO injection. As presented in Fig. 1, the ratios of HW/BW and LVW/BW, the expression of ANP mRNA (Fig. 1A-C) and the areas of collagen deposition in the interstitial and perivascular (Fig. 1D and E) were significantly increased in the ISO7d and ISO14d groups compared with the control group $(\mathrm{P}<0.05$ and $\mathrm{P}<0.01)$.

Effect of ISO on myocardial apoptosis. Cardiomyocyte apoptosis is now considered as a hallmark of heart failure (24). Using a TUNEL assay, cardiac apoptotic cells are shown by brown-stained nuclei whereas non-apoptotic cells are stained blue-green or tan shades. In the ISO7d and ISO14d groups, the apoptosis rates were significantly increased compared with the control group $(\mathrm{P}<0.05$ and $\mathrm{P}<0.01$, respectively; Fig. $2 \mathrm{~A}$ and $\mathrm{B})$. Furthermore, the ratios cleaved caspase-9/caspase- 9 was increased in the ISO3d, ISO7d and ISO14d groups, and the ratio cleaved caspase-3/caspase-3 was significantly increased in the ISO $14 \mathrm{~d}$ group compared with the control group $(\mathrm{P}<0.05$ and $\mathrm{P}<0.01$; Fig. $2 \mathrm{C}$ and $\mathrm{D})$.

Effect of ISO on MAM pathway. It is well-known that MAMs regulate physiological functions between ER and mitochondria to maintain $\mathrm{Ca}^{2+}$ flux and lipid and metabolite exchange $(25,26)$. To investigate the effect of ISO on 
A
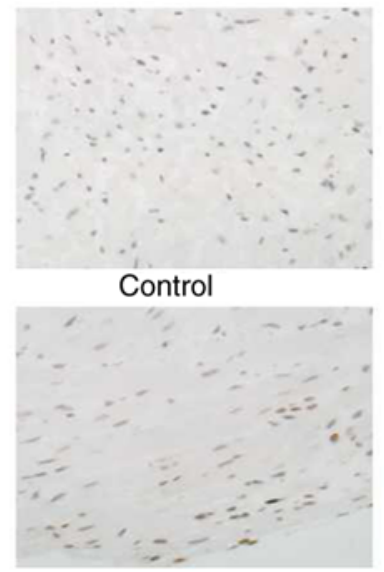

ISO 7 days
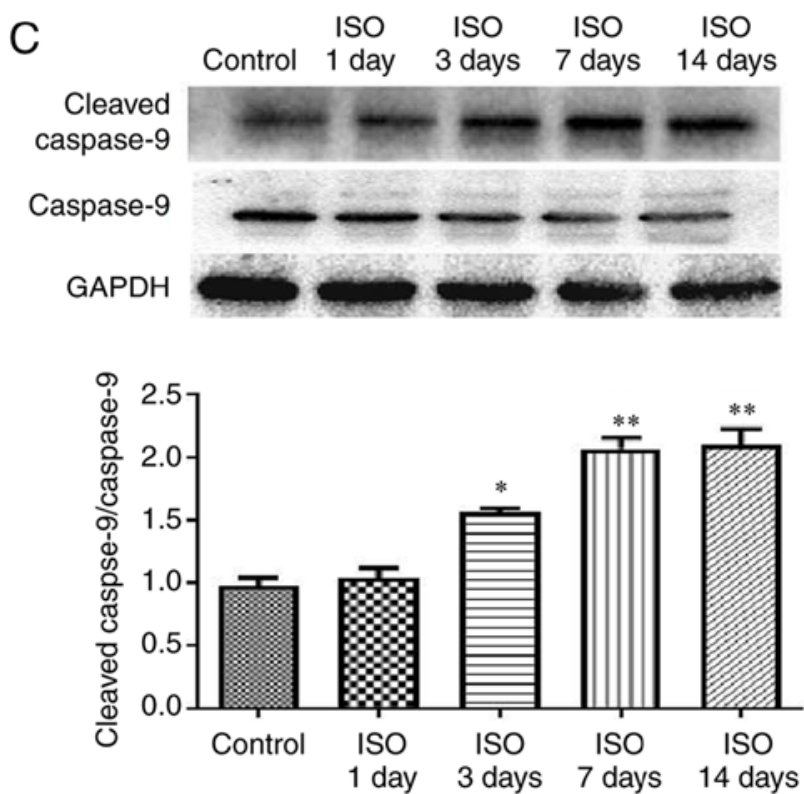

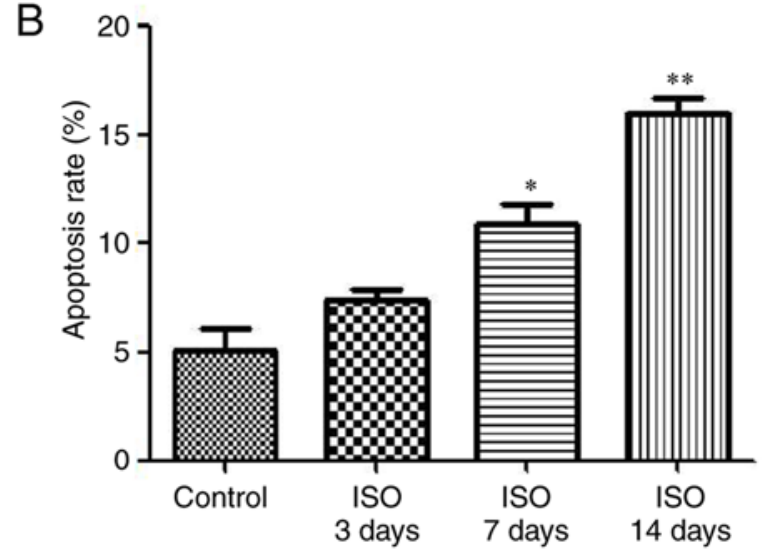

$\mathrm{D}$
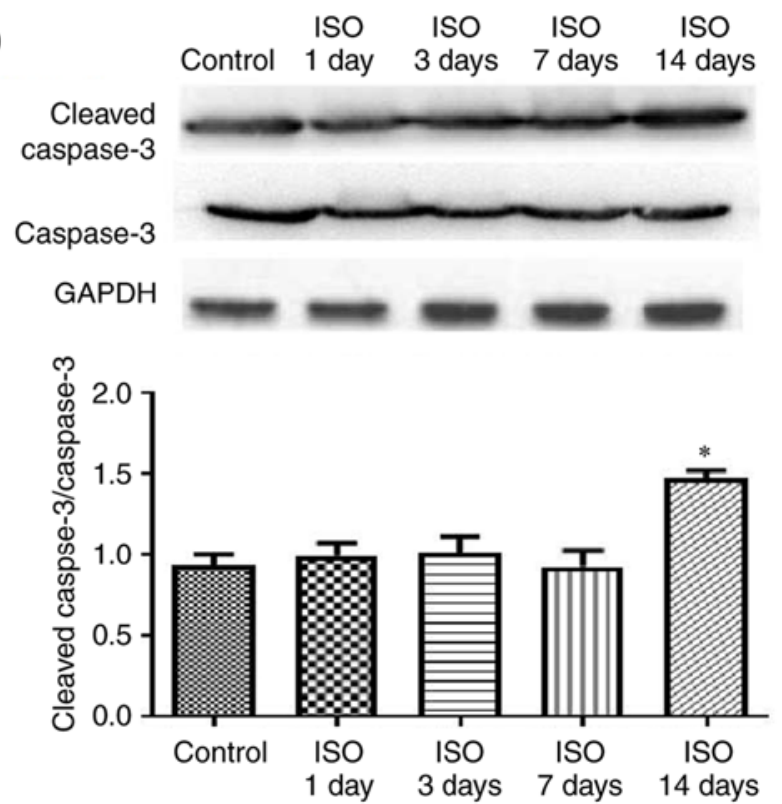

Figure 2. Effect of ISO on cardiomyocyte apoptosis in control and ISO1, 3, 7 and 14d groups. (A) TUNEL staining (brown). (B) Quantification of apoptosis rate among the various groups. (C) Protein expression of cleaved caspases-9/caspase- 9 and (D) cleaved caspases-3/caspase-3 were detected by western blotting ${ }^{*} \mathrm{P}<0.05$ and ${ }^{* *} \mathrm{P}<0.01$ vs. control group. ISO, isoproterenol.

myocardial cell MAM signal transduction, the expression of GRP75, VDAC1, Mfn2 and CypD was determined. The results demonstrated that GRP75 expression was increased in ISO14d group ( $\mathrm{P}<0.05$; Fig. 3A) whereas Mfn2 expression was unchanged in ISO groups (Fig. 3B). The protein expression of CypD was increased in ISO7d and ISO14d groups $(\mathrm{P}<0.05$; Fig. 3C) and the protein expression of VDAC 1 was increased in ISO14d group $(\mathrm{P}<0.05$; Fig. 3D) compared with control group.

DFMO alleviates myocardial hypertrophy and apoptosis induced by ISO. Cardiac hypertrophy and apoptosis were measured to assess the cytoprotective effect of DFMO. The ratios $\mathrm{HW} / \mathrm{BW}$ and $\mathrm{LVW} / \mathrm{BW}$ and the mRNA expression of ANP were significantly decreased in the DFMO treatment group compared with the ISO14d group ( $\mathrm{P}<0.05$; Fig. 4A-C). Furthermore, caspase- 3 and caspase- 9 protein expression was increased in ISO group compared with control group, which was reversed following DFMO treatment (Fig. 4D and E). In addition, the apoptosis rate was significantly decreased following DFMO treatment compared with the ISO group $(\mathrm{P}<0.05$; Fig. 4F). These findings indicated that DFMO may alleviate ISO-induced myocardial hypertrophy and myocardial apoptosis.

DFMO downregulates GRP75, VDAC1 and CypD and upregulates cardiomyocyte autophagy. The effects of DFMO on the MAM pathway and autophagy were evaluated in ISO-treated rats. The results demonstrated that in rats with ISO-induced myocardial hypertrophy, DFMO could downregulate the expression of GRP75, VDAC1 and CypD and upregulate the expression of the autophagy-associated proteins Atg5 and Beclin1, leading to an attenuation of ISO effects $(\mathrm{P}<0.05$ and $\mathrm{P}<0.01$; Fig. 5A-E). 

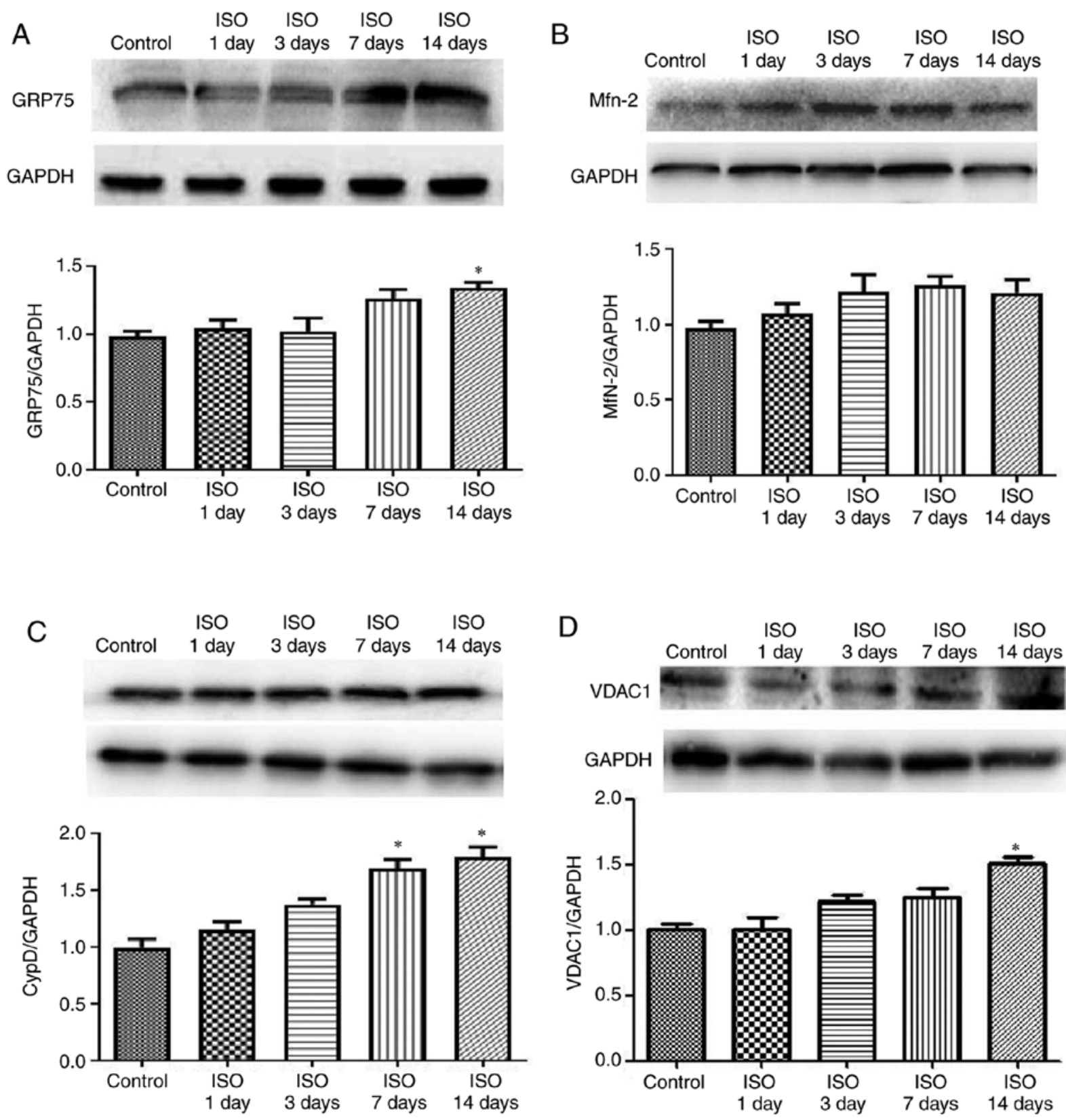

Figure 3. Effect of ISO on mitochondria-associated membranes pathway proteins in rat cardiac tissue apoptosis in control and ISO1, 3, 7 and $14 \mathrm{~d}$ groups. Protein expressions (A) of GRP75, (B) Mfn2, (C) CypD and (D) VDAC1 were detected by western blotting. "P<0.05 vs. control group. GRP75, glucose regulated protein 75; Mfn2, mitofusin2; CypD, cyclophilin D; VDAC1, voltage-dependent anion channels 1.

\section{Discussion}

The present study demonstrated that DFMO could significantly attenuate ISO-induced cardiac hypertrophy and apoptosis and downregulate the MAM pathway while increasing autophagy. It is widely known that $\beta$-adrenergic receptor activation maintains cardiac output (27), but that sustained stimulation results in heart failure $(5,28)$. The present study demonstrated that cardiac hypertrophy, fibrosis and apoptosis were induced following ISO treatment. Cardiomyocyte apoptosis is involved in the pathogenesis and progression of cardiac dysfunction during heart failures, such as myocardial infarction, $\mathrm{HF}$ and ischemia reperfusion $(26,27)$. Previous studies have suggested that increased cardiomyocyte apoptosis is implicated in the pathogenesis and development of cardiovascular complications (28-30).
Accumulating evidence showed that MAMs, including VDAC, GRP75 and CypD, are fundamental for cellular homeostasis to support mitochondrial $\mathrm{Ca}^{2+}$ signaling (31-33). Previous studies have reported that mitochondria are critical integrators of signal transduction during the development of cardiac hypertrophy $(34,35)$. It has been demonstrated that VDAC, which is associated with the outer mitochondrial membrane, interacts with IP3R on the ER through the molecular chaperone GRP75 that is thought to participate in the refolding of proteins translocated into this organelle (36). VDAC1 may be associated with both mPTP opening and mitochondrial membrane depolarization. Importantly, VDAC1 is upregulated during cardiac hypertrophy $(37,38)$. The results from the present study indicated that MAM pathway may play a key role during cardiac hypertrophy, and 

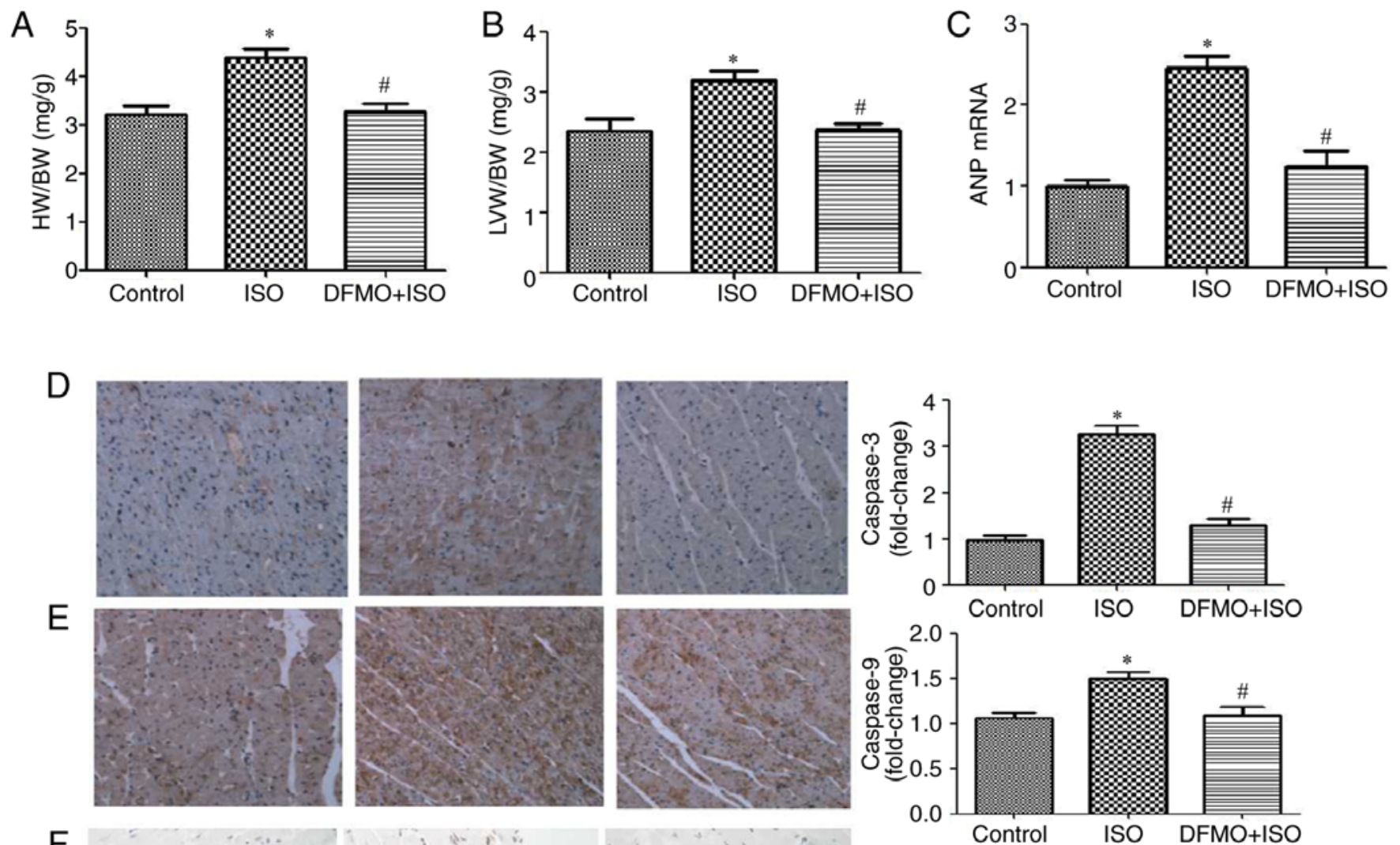

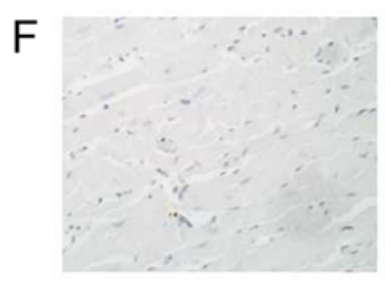

Control

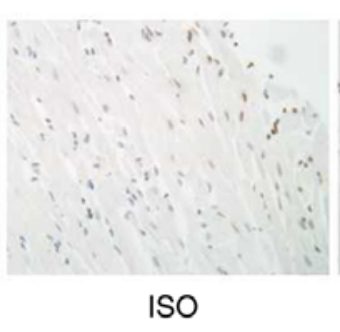

ISO

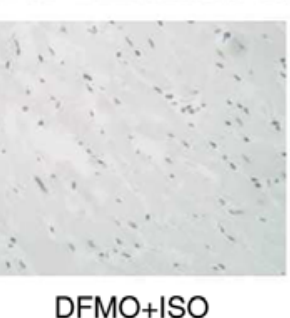

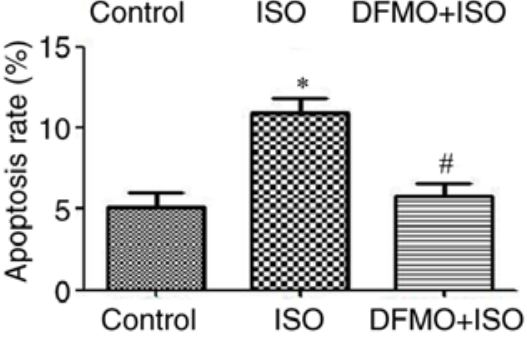

Figure 4. Effect of DFMO on cardiomyocyte hypertrophy and apoptosis induced by ISO in control, ISO and DFMO pretreatment groups. (A) HW/BW ratios. (B) LVW/BW ratios. (C) mRNA expression of ANP. (D) Immunohistological staining of caspase-3 (magnification, x200) and respective fold change. (E) Immunohistological staining of caspase-9 (magnification, $\mathrm{x} 200$ ) and respective fold change. (F) TUNEL staining (magnification, $\mathrm{x} 200$ ) and determination of the apoptosis rate. ${ }^{*} \mathrm{P}<0.05$ vs. control group. ${ }^{~} \mathrm{P}<0.05$ vs. ISO group. $\mathrm{HW} / \mathrm{BW}$, heart to body weight; LVW/BW, left ventricular to body weight; ANP, atrial natriuretic peptide; ISO, isoproterenol; DFMO, difluoromethylornithine.

that downregulation of the MAM pathway may be cardioprotective.

Polyamines (Pas) levels are tightly regulated by ODC, which is the key enzyme controlling the biosynthesis of PAs. A previous study from our group has demonstrated that DFMO could deplete cellular polyamines via inhibition of ODC (19) and attenuate cardiomyocyte hypertrophy by inhibiting the NO/cGMP-dependent protein kinase-1 pathway and ERS pathway in vivo (20). The findings from the present study indicated DFMO treatment may prevent ISO-induced cardiac hypertrophy, fibrosis and cardiomyocyte apoptosis, and downregulate the MAM pathway.

Autophagy maintains cellular homeostasis and regulates apoptosis by degrading proteins with long half-lives or damaging organelles $(39,40)$. The complexes formed by ATG5 and Beclin-1 proteins are the core molecular machinery for activation of the autophagy pathway (41). Previous studies have reported that MAMs serve a crucial role in autophagy. Beclin1 is a proautophagic protein that forms at specific regions of MAM in starvation-induced autophagy $(42,43)$. To explore the effect of DFMO on autophagy, the present study evaluated the expression of Atg5 and Beclin1. The results demonstrated that Atg5 expression was significantly decreased in ISO-induced hypertrophic rat hearts, and that DFMO could significantly increase the expression of Atg5 and Beclin1. We therefore hypothesized that Atg5 and Beclin1 may promote the survival of cardiomyocytes and inhibit hypertrophy.

In summary, the present study used an animal model of ISO-induced cardiac hypertrophy to assess the protective effect of DFMO. In ISO-treated rats, the expression of MAM pathway proteins was upregulated and associated with ISO-induced cardiac hypertrophy. Importantly, DFMO was demonstrated to ameliorate cardiac hypertrophy via inhibition of the MAM pathway and activation of the autophagy pathway (Fig. 6) One potential limitation of this study would be that the effect of DFMO on heart function, including ejection fraction and fractional shortening using echocardiography, was not evaluated. Further investigation is therefore needed to understand the relationship between 
A
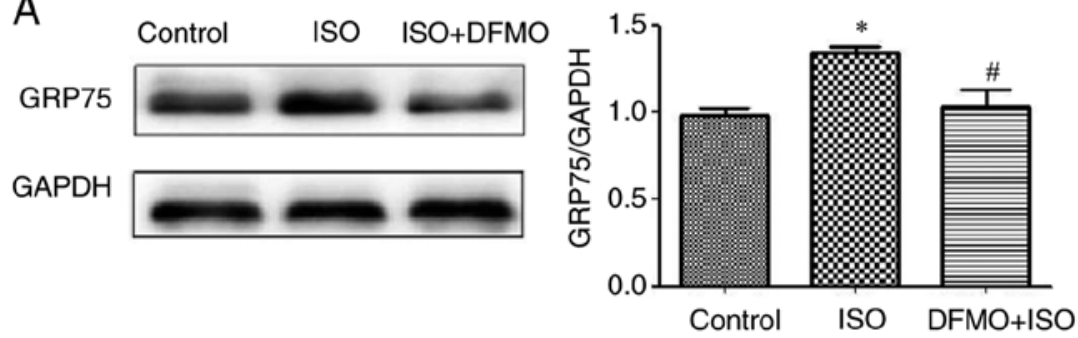

B
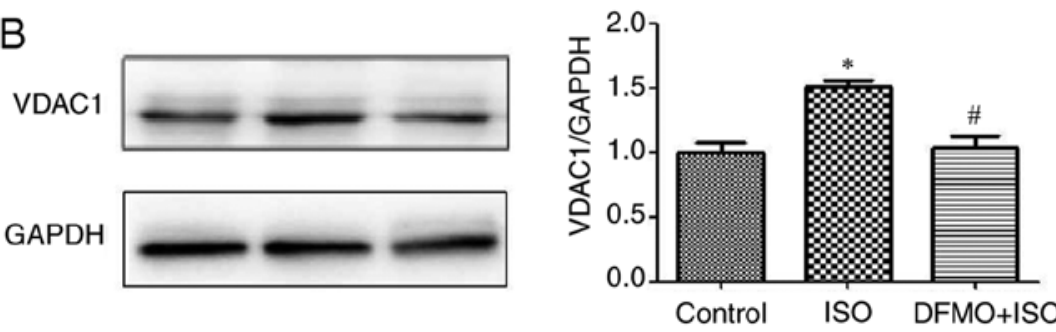

C
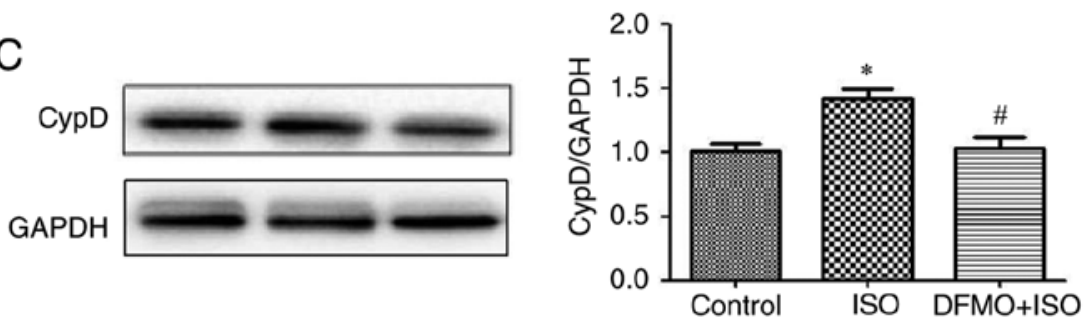
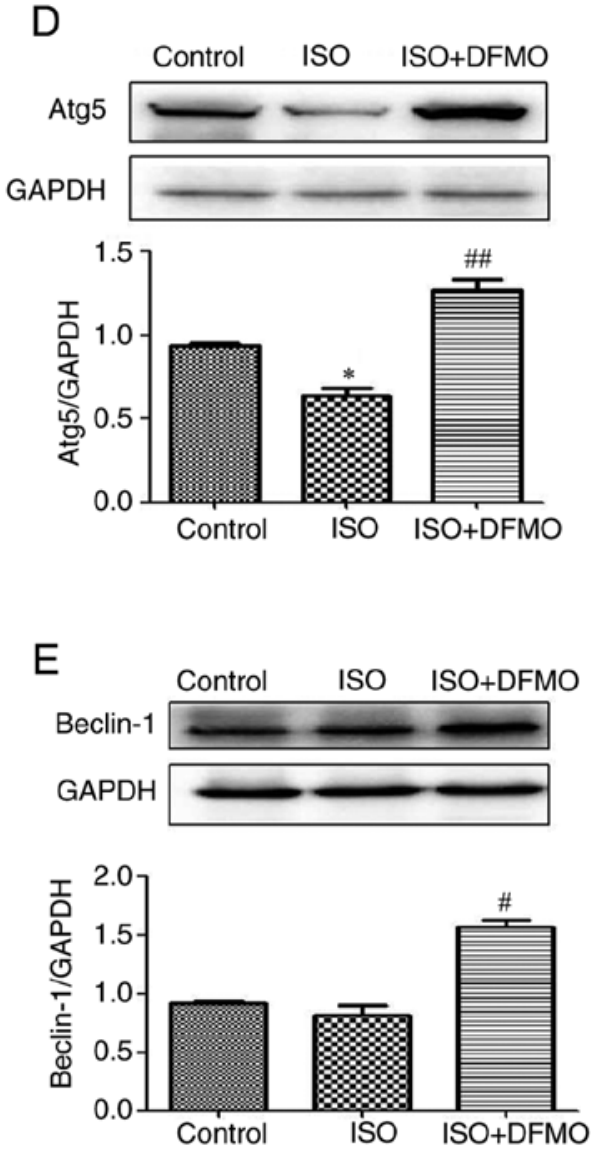

Figure 5. Effect of DFMO on mitochondria-associated membranes pathway proteins and autophagy proteins following treatment with ISO in control, ISO and DFMO pretreatment groups. Protein expression of (A) GRP75, (B) VDAC1, (C) CypD, (D) Atg5 and (E) Beclin-1 was detected by western blotting. " $\mathrm{P}<0.05$ vs. control group; ${ }^{\#} \mathrm{P}<0.05$ and ${ }^{\# \#} \mathrm{P}<0.01$ vs. ISO group. GRP75, glucose regulated protein 75; Mfn2, mitofusin2; CypD, cyclophilin D; VDAC1, voltage-dependent anion channels 1; ISO, isoproterenol; DFMO, difluoromethylornithine.

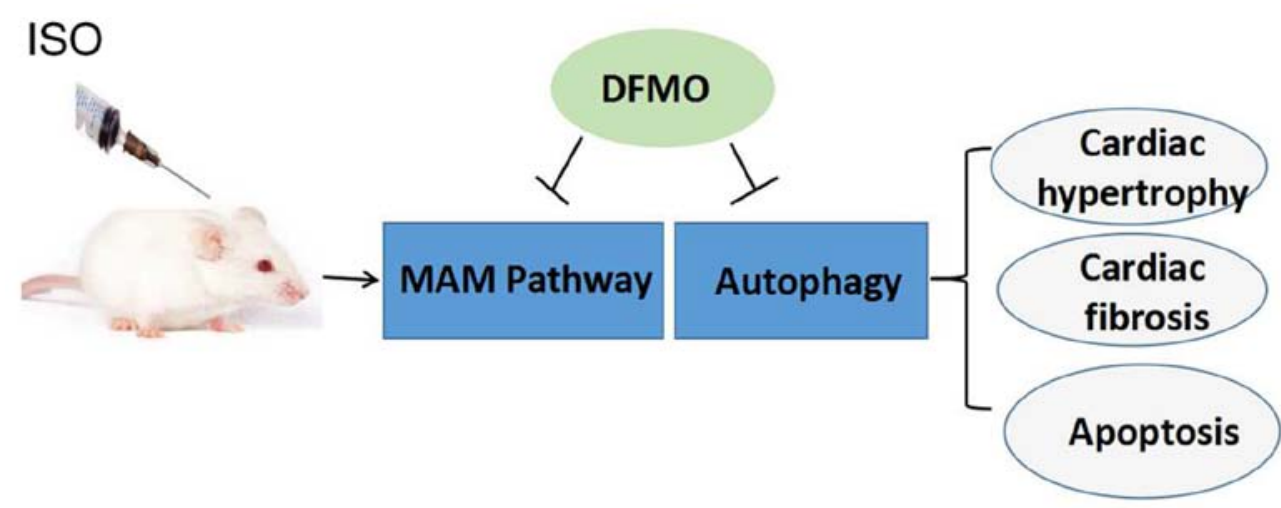

Figure 6. DFMO ameliorates cardiac hypertrophy induced by ISO via regulating autophagy and the MAM pathway in rats. DFMO, difluoromethylornithine. ISO, isoproterenol; MAM, mitochondria-associated membranes.

MAM and the cardiac function following treatment with DFMO. The findings from the present study suggested that DFMO may be considered as a potential therapeutic agent to treat cardiac hypertrophy.

\section{Acknowledgements}

Not applicable.

\section{Funding}

This study was supported by the National Natural Science Foundation of China (grant nos. 82074148, 31751004), the Natural Science Foundation of Heilongjiang Province (grant no. H2017080) and the Postdoctoral Scientific Research Foundation of Heilongjiang Province (grant no. LBH-Q18128). 


\section{Availability of data and materials}

The datasets used and/or analyzed during the present study are available from the corresponding author on reasonable request.

\section{Authors' contributions}

YZ and YL designed the experiments and wrote and revised the manuscript. WWJ, SR and WX performed the experiments and analyzed the data. GWL and LJ carried out the experiments and revised the manuscript. All authors read and approved the final version. YL and GWL confirm the authenticity of all the raw data.

\section{Ethics approval and consent to participate}

The experimental protocols were approved by the Committee on the Ethics of Animal Experiments of Qiqihar Medical University, China (approval no. QMU-AECC-2019-53).

\section{Patient consent for publication}

Not applicable.

\section{Competing interests}

The authors declare that they have no competing interests.

\section{References}

1. Branco AF, Sampaio SF, Wieckowski MR, Sardão VA and Oliveira PJ: Mitochondrial disruption occurs downstream from - adrenergic overactivation by isoproterenol in differentiated, but not undifferentiated H9c2 cardiomyoblasts: Differential activation of stress and survival pathways. Int J Biochem Cell Biol 45: 2379-2391, 2013.

2. Berenji K, Drazner MH, Rothermel BA and Hill JA: Does load-induced ventricular hypertrophy progress to systolic heart failure? Am J Physiol Heart Circ Physiol 289: H8-H16, 2005.

3. Forte M, Schirone L, Ameri P, Basso C, Catalucci D, Modica J, Chimenti C, Crotti L, Frati G, Rubattu S, et al: The role of mitochondrial dynamics in cardiovascular diseases. Br J Pharmacol 15: 1-17, 2020.

4. Wyss RK, Méndez-Carmona N, Sanz MN, Arnold M, Segiser A, Fiedler GM, Carrel TP, Djafarzadeh S, Tevaearai Stahel HT and Longnus SL: Mitochondrial integrity during early reperfusion in an isolated rat heart model of donation after circulatory death-consequences of ischemic duration. J Heart Lung Transplant 38: 647-657, 2019.

5. Dudek J, Hartmann M and Rehling P: The role of mitochondrial cardiolipin in heart function and its implication in cardiac disease. Biochim Biophys Acta Mol Basis Dis 1865: 810-821, 2019.

6. Raut GK, Manchineela S, Chakrabarti M, Bhukya CK, Naini R, Venkateshwari A, Reddy VD,Mendonza JJ,Suresh Y,NallariP, etal: Imine stilbene analog ameliorate isoproterenol-induced cardiac hypertrophy and hydrogen peroxide-induced apoptosis. Free Radic Biol Med 153: 80-88, 2020.

7. Wang S, Binder P, Fang Q, Wang Z, Xiao W, Liu W and Wang X: Endoplasmic reticulum stress in the heart: Insights into mechanisms and drug targets. Br J Pharmacol 175: 1293-1304, 2018.

8. Rieusset J: Mitochondria and endoplasmic reticulum: Mitochondria-endoplasmic reticulum interplay in type 2 diabetes pathophysiology. Int J Biochem Cell Biol 43: 1257-1262, 2011.

9. Monteiro JP, Oliveira PJ and Jurado AS: Mitochondrial membrane lipid remodeling in pathophysiology: A new target for diet and therapeutic interventions. Prog Lipid Res 52: 513-528, 2013.

10. Tang LL, Wang JD, Xu TT, Zhao Z, Zheng JJ, Ge RS and Zhu DY: Mitochondrial toxicity of perfluorooctane sulfonate in mouse embryonic stem cell-derived cardiomyocytes. Toxicology 382 : 108-116, 2017.
11. Sironi L, Restelli LM, Tolnay M, Neutzner A and Frank S: Dysregulated interorganellar crosstalk of mitochondria in the pathogenesis of Parkinson's disease. Cells 9: 233, 2020.

12. Murata D, Arai K, Iijima M and Sesaki H: Mitochondrial division, fusion and degradation. J Biochem 167: 233-241, 2020.

13. Ou Y, Wang SJ, Li D, Chu B and Gu W: Activation of SAT1 engages polyamine metabolism with $\mathrm{p} 53$-mediated ferroptotic responses. Proc Natl Acad Sci USA 113: E6806-E6812, 2016.

14. Pegg AE: Functions of polyamines in mammals. J Biol Chem 291: 14904-14912, 2016.

15. Murray Stewart T, Dunston TT, Woster PM and Casero RA Jr: Polyamine catabolism and oxidative damage. J Biol Chem 293: 18736-18745, 2018.

16. Chen M, Xin J, Liu B, Luo L, Li J, Yin W and Li M: Mitogen-activated protein kinase and intracellular polyamine signaling is involved in TRPV1 activation-induced cardiac hypertrophy. J Am Heart Assoc 5: e003718, 2016.

17. Lin Y, Zhang X, Wang L, Zhao Y, Li H, Xiao W, Xu C and Liu J: Polyamine depletion attenuates isoproterenol-induced hypertrophy and endoplasmic reticulum stress in cardiomyocytes. Cell Physiol Biochem 34: 1455-1465, 2014.

18. Casero RA Jr, Murray Stewart T and Pegg AE: Polyamine metabolism and cancer: Treatments, challenges and opportunities. Nat Rev Cancer 18: 681-695, 2018.

19. Lin Y, Liu JC, Zhang XJ, Li GW, Wang LN, Xi YH, Li HZ, Zhao YJ and Xu CQ: Downregulation of the ornithine decarboxylase/polyamine system inhibits angiotensin-induced hypertrophy of cardiomyocytes through the $\mathrm{NO} /$ cGMP-dependent protein kinase type-I pathway. Cell Physiol Biochem 25: 443-450, 2010.

20. Pegg AE: Regulation of ornithine decarboxylase. J Biol Chem 281: 14529-14532, 2006.

21. Lin Y, Zhang X, Xiao W, Li B, Wang J, Jin L, Lian J, Zhou L and Liu J: Endoplasmic reticulum stress is involved in DFMO attenuating isoproterenol-induced cardiac hypertrophy in rats. Cell Physiol Biochem 38: 1553-1562, 2016.

22. Jiang G, Gong H, Niu Y, Yang C, Wang S, Chen Z, Ye Y, Zhou N, Zhang $\mathrm{G}, \mathrm{Ge} \mathrm{J}$, et al: Identification of amino acid residues in angiotensin ii type 1 receptor sensing mechanical stretch and function in cardiomyocyte hypertrophy. Cell Physiol Biochem 37: 105-116, 2015.

23. Wang W, Zhang H, Xue G, Zhang L, Zhang W, Wang L, Lu F, Li H, Bai S, Lin Y, et al: Exercise training preserves ischemic preconditioning in aged rat hearts by restoring the myocardial polyamine pool. Oxid Med Cell Longev 2014: 457429, 2014.

24. Braunwald E: Heart failure. JACC Heart Fail 1: 1-20, 2013.

25. Perrone M, Caroccia N, Genovese I, Missiroli S, Modesti L, Pedriali G, Vezzani B, Vitto VAM, Antenori M, Lebiedzinsk a-Arciszewska $\mathrm{M}$, et al: The role of mitochondria-associated membranes in cellular homeostasis and diseases. Int Rev Cell Mol Biol 350: 119-196, 2020.

26. Imaeda A, Tanaka S, Tonegawa K, Fuchigami S, Obana M, Maeda M, Kihara M, Kiyonari H, Conway SJ, Fujio Y, et al: Myofibroblast $\beta 2$ adrenergic signaling amplifies cardiac hypertrophy in mice. Biochem Biophys Res Commun 510: 149-155, 2019.

27. de Lucia C, Eguchi A and Koch WJ: New insights in cardiac $\beta$-adrenergic signaling during heart failure and aging. Front Pharmacol 9: 904, 2018.

28. Ferrara N, Komici K, Corbi G, Pagano G, Furgi G, Rengo C, Femminella GD, Leosco D and Bonaduce D: $\beta$-adrenergic receptor responsiveness in aging heart and clinical implications. Front Physiol 4: 396, 2014.

29. Ramani D, De Bandt JP and Cynober L: Aliphatic polyamines in physiology and diseases. Clin Nutr 33: 14-22, 2014.

30. Li Y and Liu X: The inhibitory role of Chinese materia medica in cardiomyocyte apoptosis and underlying molecular mechanism. Biomed Pharmacother 118: 109372, 2019.

31. Huang P, Fu J, Chen L, Ju C, Wu K, Liu H, Liu Y, Qi B, Qi B and Liu L: Redd1 protects against post infarction cardiac dysfunction by targeting apoptosis and autophagy. Int $\mathrm{J}$ Mol Med 44: 2065-2076, 2019.

32. Takeshima H, Venturi E and Sitsapesan R: Takeshima1 H, Venturi E and Sitsapesan R: New and notable ion-channels in the sarcoplasmic/endoplasmic reticulum: Do they support the process of intracellular $\mathrm{Ca}^{2+}$ release? J Physiol 593: 3241-3251, 2015.

33. Sun D, Chen X, Gu G, Wang J and Zhang J: Potential roles of mitochondria-associated ER membranes (MAMs) in traumatic brain injury. Cell Mol Neurobiol 37: 1349-1357, 2017. 
34. Dingreville F,Panthu B, Thivolet C,Ducreux S, Gouriou Y,Pesenti S, Chauvin MA, Chikh K, Errazuriz-Cerda E, Van Coppenolle F, et al: Differential effect of glucose on ER-mitochondria $\mathrm{Ca}^{2+}$ exchange participates in insulin secretion and glucotoxicity-mediated dysfunction of $\beta$-cells. Diabetes 68: 1778-1794, 2019.

35. Betz C, Stracka D, Prescianotto-Baschong C, Frieden M, Demaurex N and Hall MN: mTOR complex 2-Akt signaling at mitochondria-associated endoplasmic reticulum membranes (MAM) regulates mitochondrial physiology. Proc Natl Acad Sci USA 110: 12526-12534, 2013.

36. Roman B, Kaur P, Ashok D, Kohr M, Biswas R, O'Rourke B, Steenbergen $C$ and Das sS: Nuclear-mitochondrial communication involving miR-181c plays an important role in cardiac dysfunction during obesity. J Mol Cell Cardiol 144: 87-96, 2020.

37. Szabadkai G, Bianchi K, Várnai P, De Stefani D, Wieckowski MR, Cavagna D, Nagy AI, Balla T and Rizzuto R: Chaperone-mediated coupling of endoplasmic reticulum and mitochondrial $\mathrm{Ca}^{2+}$ channels. J Cell Biol 175: 901-911, 2006.

38. Mitra A, Basak T, Datta K, Naskar S, Sengupta S and Sarkar S: Role of $\alpha$-crystallin $\mathrm{B}$ as a regulatory switch in modulating cardiomyocyte apoptosis by mitochondria or endoplasmic reticulum during cardiac hypertrophy and myocardial infarction. Cell Death Dis 4: e582, 2013.
39. Javadov S, Rajapurohitam V, Kilić A, Zeidan A, Choi A and Karmazyn M: Anti-hypertrophic effect of NHE-1 inhibition involves GSK-3beta-dependent attenuation of mitochondrial dysfunction. J Mol Cell Cardiol 46: 998-1007, 2009.

40. Li X, Lu J, Xu Y, Wang J, Qiu X, Fan L, Li B, Liu W, Mao F, Zhu J, et al: Discovery of nitazoxanide-based derivatives as autophagy activators for the treatment of Alzheimer's disease. Acta Pharm Sin B 10: 646-666, 2020.

41. Cheon SY, Kim H, Rubinsztein DC and Lee JE: Autophagy, cellular aging and age-related human diseases. Exp Neurobiol 28: 643-657, 2019.

42. Mizushima N, Yoshimori T and Ohsumi Y: The role of Atg proteins in autophagosome formation. Annu Rev Cell Dev Biol 27: 107-132, 2011.

43. Gelmetti V, De Rosa P, Torosantucci L, Marini ES, Romagnoli A, Di Rienzo M, Arena G, Vignone D, Fimia GM and Valente EM: PINK1 and BECN1 relocalize at mitochondria-associated membranes during mitophagy and promote ER-mitochondria tethering and autophagosome formation. Autophagy 13: 654-669, 2017.

This work is licensed under a Creative Commons Attribution-NonCommercial-NoDerivatives 4.0 International (CC BY-NC-ND 4.0) License. 